\title{
A Dutch report on the ethics of neonatal care
}

\author{
Zier Versluys and Richard de Leeuw Wilhelmina Children's Hospital, Utrecht, Holland and AMC/Emma \\ Children's Hospital, Amsterdam, Holland, respectively
}

\begin{abstract}
The Dutch Paediatric Association reports consensus among its members regarding the necessity to take the future quality of life into account when reaching decisions regarding the continuation or dis-continuation of life-prolonging treatment. The paramount importance of the discussion with the parents is stressed. Dissension exists regarding active euthanasia in the newborn, both opinions being respected. If dissension exists within the profession parents should be informed and if necessary referred to a doctor who shares their moral views.
\end{abstract}

\section{Introduction}

After many years of discussion a report on the moral aspects of neonatal care, in particular euthanasia, was unanimously accepted by the Dutch Paediatric Association on 5 November 1992. This report has received the name 'Doen of Laten?' (To Do or Not To Do? (1). Although, as was expected from ensuing lengthy discussions, considerable consensus among paediatricians resulted, the report being very much accepted in these circles, there has been criticism from legal experts and politicians, mainly focusing on the quality-of-life decisions made by parents and doctors regarding newborns. Some church leaders have also protested along these lines. Considerable support has been found amongst Dutch ethicists, medical societies, the media and the three combined Protestant churches. The paediatricians have been widely complimented on their openness. In line with our own dissensus, active euthanasia is condemned by some and accepted as an ultimate possibility by others. The present paper aims primarily to present the discussions of the Dutch Paediatric Association, which ranged from full consensus to straight disagreement. The authors take responsibility for this summary being in line with the report, which was the result of a group process.

During the last few decades there has been an unprecedented increase in the application of

\section{Key words}

Newborn; life-prolonging treatment; euthanasia; Dutch Paediatric Association; ethics. potentially life-prolonging medical treatment to very $\stackrel{\omega}{\stackrel{\omega}{S}}$ small and very sick neonates. From the moral point $\overline{3}$ of view one should justify not only stopping ${ }_{N}^{\mathbb{D}}$ treatment in certain circumstances but also starting.or continuing treatment. This does not apply only to $\overrightarrow{-}$ very advanced medical care such as artificial $\vec{A}$ ventilation or extracorporeal oxygenation but also to은 other types of care such as gavage feeding (feeding through a naso-gastric tube), which are in fact life- $T$ (D prolonging measures. Such care is justified only as long as there is a reasonable hope of survival with a chance of an acceptable form of life.

\section{Discontinuation of life-prolonging treatment}

There is no case for life-prolonging treatment when there is no chance of survival. This is a medical decision. It must be accompanied by humane $\stackrel{\odot}{\Phi}$ support during the ensuing process of dying. This $\underset{\vec{F}}{\overrightarrow{2}}$ may include administration of medication which $\frac{9}{3}$ relieves suffering but hastens death. Actions like $\supset$ these in the terminal phase have always been part of the merciful practice of medicine and are not considered as euthanasia.

When treatment offers a prospect of survival, but: there are doubts about the quality of later life, $\underline{3}$. decision-making is much more difficult and is noto confined to the medical profession. Questions arise about the worth of the life which is to be saved ando whether the child and its family will benefit. The conclusion of the report is that the choice between life and death belongs to the area of responsibility of $\mathrm{N}$ the paediatrician in these situations. In the following ( passages the neonates in question have very severe $N$ handicaps but, nevertheless, have a chance of $\mathrm{E}_{\mathrm{E}}$ surviving 'thanks to' life-prolonging medicalo treatment. It is not a question of whether or not weo choose, because either continuing or stopping $\widetilde{\Phi}$ treatment means that a choice is being made: the $\stackrel{?}{+}$ question is how openly, how thoroughly and how humanely we make the choice.

The choice which is made should be based on a diagnosis which is meticulously arrived at using state $\overrightarrow{\mathbb{D}}$ of the art medical means. The prognosis is derivedo from this with utmost care. It is only in cases where 
a very bad prognosis is reasonably certain that there is any debate about medical actions which prolong life. However, decisions have to be made even though complete certainty cannot be attained. If one waits for absolute certainty it means that, in practice, treatment is never stopped but is continued indefinitely.

The sense (or lack of it) of administering certain forms of treatment may be questioned by doctors, nurses or parents. It is of the utmost importance that the parents and nursing staff (and not only the doctors) are fully aware of the prognosis. This can almost always be achieved when it is attempted. The prognosis should be evaluated in functional terms as much as possible. The following questions must be asked:

- What communicative abilities will the child have later?

- Will the child be able to lead an independent life?

- Will the child be continuously dependent on medical facilities?

- Will the child suffer either mentally or physically?

- How long is the life expectancy?

This information should provide the doctors and parents with an impression of the weight of the burden which a severe handicap involves. The most difficult and most subjective step comes next in measuring this information against the ability of the families and children to bear the burden. However, the necessary choice can be made only from this starting point. This can succeed only when the parents receive adequate counselling and everyone is aware that the choice must be made together. It is also essential that the available choices are made completely clear.

An example of such a situation occurs when severe cerebral damage is diagnosed in a preterm infant who is being artificially ventilated. The choices then available are the following:

\section{Continue maximal intensive care and extend it if} necessary.

2. Maintain the status quo.

3. Stop the most intensive form of treatment (for example, artificial ventilation).

4. Stop all life-prolonging measures. If suffering occurs or can be foreseen give medication to soften and hasten death.

- The first option will be chosen when there is a good prognosis.

- The second somewhat ambivalent option will be considered acceptable only as a temporary measure.

- The third option can be the best choice if this directly leads to the death of a neonate with anticipated severe handicap. Up to this point consensus exists within the Dutch Paediatric Association.

- The fourth option is acceptable to some Dutch paediatricians in certain circumstances.
An example of such circumstances is provided by the situation of a severely handicapped neonate who after being disconnected from the ventilator could possibly survive when nursed in an incubator and given gavage feeding or treatment other than intensive care treatment. In such a case some paediatricians would consider continuation of this care morally unjustified because they consider it irresponsible - non-beneficial and/or positively harmful - to prolong life in such a case. They would instead administer medication to soften and hasten the now inevitable death, arguing that without the life-support the child is in a terminal phase. This view is considered morally questionable by other Dutch paediatricians because death is caused directly by the medication. They would theoretically rather accept continuation of part of the life-prolonging treatment (although not being considered of benefit to the child) as the lesser evil. All agree that if the discontinuation of life-prolonging treatment leads to a lengthy and (although we can but guess what the child itself feels) disgusting process of dying, this cannot be accepted on humane grounds. In practice therefore in all neonatal centres artificial ventilation in these cases is sometimes discontinued in such a way that a peaceful death is achieved.

At this stage it is clear that there is some moral disagreement; this is more obvious in the case of deliberate termination of life (active euthanasia), which will be dealt with later.

No legal consequences were until very recently to be expected in the Netherlands in the aforementioned situation where a choice was made to abstain from life-prolonging actions. The infant was considered to have died from a lethal illness and a certificate of natural death was signed by the doctor in charge. A choice to abstain from using lifeprolonging measures is made hundreds of times every year in the Netherlands. However, the new Dutch law on euthanasia can be interpreted as prohibiting the issuing of a certificate of natural death if poor (future) quality of life was a major reason for abstaining from treatment.

\section{Starting life-prolonging treatment}

So much for the discontinuation of life-prolonging treatment once started. Theoretically we do not consider the moral considerations regarding stopping treatment to be very different from those regarding starting it. In the practice of neonatology, however, thorough decisions can often not be made in the first minutes and hours of extra-uterine life. Yet at the start of life it is minutes that count. Therefore in general we implement all available intensive care measures in order to prolong life, with evaluation as soon as possible: 'act first think later'. The decision to start treatment without a clear view on the harm/benefit ratio should be considered as 'provisional treatment'. But, like any general policy, 
this one has its limits. For instance, while treating extremely preterm babies below 26 weeks' gestational age, with a high risk of severe handicaps, prolonged periods of inpatient care, considerable late mortality and in view of our actual reluctance to stop once started, we have to question the wisdom of this policy.

\section{Active euthanasia in the newborn}

An even more difficult moral choice has to be made about ten times a year in the cases of neonates who, likely to be severely handicapped, may survive without any life-prolonging medical care. This implies that they are able to drink and can be cared for in an ordinary cot. One example of such a case is that of a neonate who has been previously kept alive with the aid of advanced medical care, but in whom serious cerebral damage has become apparent. Another example is that of a neonate with such severe congenital malformations that surgical treatment was withheld, but the infant nevertheless remained alive. It is in these extremely serious situations that some Dutch paediatricians regard active euthanasia (by administration of medication) to be morally justifiable. They argue that in some extreme circumstances the doctor, who often has given life-prolonging treatment before or will give it later, can morally be allowed, or even obliged as an act of mercy, to 'give death' to a newborn for whom life would be an agony. Mercy (misericordia) is the source of medicine and its reason for being.

However, other paediatricians consider this course of action morally unjustifiable. Doctors, they argue, are responsible for the treatment they give and should withhold treatment if it does more harm than good; purposely and actively taking the life of a newborn (not in a terminal phase) is beyond the scope of medicine and a transgression of our human moral borders.

These two standpoints, matters of principle, were unlikely to be resolved in an easy compromise, certainly not within the time we as practical doctors planned to take for our report. Still it is worthwhile to outline the existing dissension. Also we could agree that within our paediatric association both points of view were respected and that parents had the right to know about this dissension and to know where their doctor stood in this respect.

From the legal point of view deliberate termination of life means that the death was caused by the doctor. It appears therefore that these cases should, under the new Dutch law on euthanasia, bঙ reported to the prosecuting attorney. So far no one has been prosecuted. However, the legal situation ist. far from clear - and it requires quite some courage te? report such a case.

\section{Decision-making}

In view of the differences which exist in a multiracias and multicultural society it is important that suck decisions concerning the quality of later life are taken by all the staff as a team. The parents' wishes. are very important and when the attending doctor cannot support these wishes the parents should be given the opportunity to choose another doctor. It i. essential that the parents receive adequate counselling and support, including sufficienfo information and ample time for consideration and that they have a good personal relationship with the attending doctor. However, even though teanf consultation and a continuous dialogue with the parents are of extreme importance, it is the attending doctor who remains responsible for the chosem policy.

The decisions discussed here must be evaluate and discussed within hospitals. A copy of the Dutch Paediatric Association report Doen of Laten (1) (or 8 summary) should be available for perusal in evergr neonatal department in the Netherlands. It showid also be available to parents. Nursing or medical who have moral objections to the policy chosen $\%$ the parents or the team should have the right to bo excused from participating. Polarized moral view which cannot be resolved at department level mus be laid before the hospital's medical ethic committee or the hospital board.

Zier Versluys, PhD, is a Paediatrician-Neonatologist. working in the Wilhelmina Children's Hospital, Utrecht Holland. Richard de Leeuw is a Paediatriciane Neonatologist, working in the AMC/Emma Children Hospital, Amsterdam, Holland.

\section{Note}

(1) This report was prepared by a working grout consisting of $\mathrm{Z}$ Versluys (chairman), $\mathrm{R}$ de Leeuw: (secretary), F H M Jansen, L A A Kollée, P J J Saueŝ and $\mathrm{H}$ Wieringa. It includes a short summary ip English and is available from the Dutch Paediatrio Association, Postbus 20059, 3502 LB Utrecht, the Netherlands. 